\title{
Occurrence and cytological mechanism of numerically unreduced pollen in diploid Populus euphratica
}

\author{
By Pingdong ZHANG ${ }^{1)}$ and XIANGYANG KAnG $^{1), *}$
}

(Received $4^{\text {th }}$ February 2013)

\begin{abstract}
Unreduced gametes are the driving force for the polyploidizaiton of plants in nature, and are also an important tool for breeding of triploid individuals. The final heterozygosity of a $2 \mathrm{n}$ pollen grain depends on the cytological mechanism behind $2 \mathrm{n}$ pollen formation. In this study, meiotic abnormalities were analysed using fluorescent chromosome staining and indirect immunofluorescence during the microsporogenesis of 18 genotypes of diploid $P$. euphratica Oliv. $(2 \mathrm{n}=2 \mathrm{x}=38)$. Among the 18 genotypes, 16 genotypes produce $2 \mathrm{n}$ pollen and two genotypes produce only normal $\mathrm{n}$ pollen. In all $2 \mathrm{n}$ pollen producers, we found that the first meiotic division was normal but that the second division was characterized by frequent abnormal spindle orientation (parallel, tripolar, and fused spindles) and premature cytokinesis. The parallel, fused spindles and premature cytokinesis were considered to be leading dyad formation, and tripolar spindles seemed to be causing triad formation at the tetrad stage. There was a higher frequency of parallel spindles than other spindle forms, but no significant correlations between parallel spindles and dyads were observed. However, a significant association ( $r=0.68$, $P<0.05$ ) between the tripolar spindles and dyads was found. In some Microspore mother cells (MMCs), an indirect immunofluorescence examination of meiosis II revealed that the parallel spindles led to the gathering of one or two non-sister groups of chromosomes, causing an incorporation of RMSs from two daughter nuclei. Therefore, the incorporated RMSs established two nuclear cytoplasmic domains for the control of division plane, resulting in either triad or dyad formation.
\end{abstract}

Key words: Populus euphratica, Unreduced pollen, Spindle orientation, Cytokinesis, Triploid breeding.

\section{Introduction}

In plant species and hybrids, normal diploid $(2 \mathrm{n}=2 \mathrm{x})$ plants produce genetically four different male gametes $(\mathrm{n}, \mathrm{n}, \mathrm{n}, \mathrm{n})$ through complex pathways of microsporogenesis. Numerically unreduced (2n) gametes occur occasionally in stead of the expected haploid (n) gametes and likely played a key role in formation of polyploid series (HARlAN and De Wet, 1975; Den NiJs and PELOQUin, 1977; VeIlleux, 1985; RAMSEY and Schemske, 1998; RAMSEY, 2007; RIESEBERG and Willis, 2007). The induction of sexual polyploidization via controlled crosses

\footnotetext{
1) National Engineering Laboratory for Tree Breeding, Key laboratory of Genetics and Breeding in Forest Trees and Ornamental Plants, Ministry of Education, Beijing Forestry University, No. 35, Qinghua East Road, Haidian District, 100083 Beijing, People's Republic of China.

*) Corresponding author: Xiangyang Kang. Tel +86-010-62336168; Fax +86-010-62336168. E-Mail: Kangy@ @bju.edu.cn
}

between naturally occurring and aritificially induced-2n gametes have also been widely used in the breeding of forest trees (SEITZ, 1954; ZHANG et al., 2004; ZHU, 2006), potato (MENDIBURU et al., 1974; RAMANNA, 1979; CARPUTO and BARONE, 2005), grain crops (VeIlleuX, 1985; JAUHAR, 2003), fruit-bearing trees (SUGIURA et al., 2000), and ornamental plants (VAN TUYL and LIM, 2003; AkUTSU et al., 2007).

The mechanisms of $2 \mathrm{n}$ gamete formation have been studied in numerous plant species (VEILlEUX, 1985; BRETAGNOLle and THOMPSON, 1995; RAMSEY and SCHEMSKE, 1998), and various possible pathways causing the formation of $2 \mathrm{n}$ gametes have been revealed: chromosome pairing, centromere division, spindle orientation, abnormal cytokinesis, post-meiotic doubling, and apomeiotic cells of the ovule. However, the most accepted currently held opinion is that $2 \mathrm{n}$ gamete formation is the result of irregular orientations of spindles and abnormal cytokinesis at the second meiotic division (Veilleux, 1985; Bretagnolle and Thompson, 1995; RAMSEY and SCHEMSKe, 1998; ZhaNG et al., 2009). Disturbed spindles and cytokinesis related to $2 \mathrm{n}$ pollen formation have been reported to be associated with changes in the configurations of the microtubules (GENUALDO et al., 1998; CoNiCELle et al., 2003; ZHANG et al., 2009; ZHANG and KANG, 2010). In addition, D'ERFURTH et al. (2008) recently isolated and characterized a gene (AtPSl) involved in the abnormal orientation of spindles at meiosis II that apparently controlled diploid ( $2 \mathrm{n}$ ) gamete formation in Arabidopsis thaliana.

The genetic constitution of $2 \mathrm{n}$ gametes generally divided into three categories, first division restitution (FDR), second division restitution (SDR) and indeterminate meiotic restitution (IMR). A FDR $2 n$ gamete contains non-sister chromatids, while a SDR 2n gamete contains two sister chromatids (TANG, 2002). However, the third (IMR) type, numerically disproportionate numbers of chromosomes occur due to a restitution mechanism which cannot be categorised as either FDRor SDR. This restitution mechanism was detected in interspecific lily hybrids (LIM et al., 2001), and showed characteristics similar to both SDR and FDR. In this intermediate type, both univalents and bivalents are formed during metaphase I.

In the genus Populus, unreduced pollen has been documented in $P$. canescens Moench. $(2 \mathrm{n}=2 \mathrm{x}=38)$ (Seitz, 1954), P. nigra L. $(2 \mathrm{n}=2 \mathrm{x}=38)($ BraDSHAW and Stettler, 1993), $P$. tomentosa Carr. $(2 \mathrm{n}=2 \mathrm{x}=38)$ (ZHU et al., 1998; ZHANG et al., 2007; ZHANG and KANG, 2010), P. tremula L. $(2 \mathrm{n}=2 \mathrm{x}=38)$ (MuntzING, 1936), $P$. $\times$ euramericana (Dode) Guinier and $P . \times$ popularis 
(ZHANG et al., 2009). However, little is known about the rates and cytological mechanism of numerically unreduced pollen in diploid Populus euphratica in China. Understanding of the cytological mechanism of $2 \mathrm{n}$ gamete formation is vital in their utilization in breeding because genetic consequences are different in terms of the transmission of parental heterozygosity and epistasis retained in the two types of $2 n$ gametes. It has been calculated that a $2 \mathrm{n}$ gamete derived from FDR with crossing over will transmit roughly $80 \%$ of parental heterozygosity to its progeny while a SDR $2 \mathrm{n}$ gamete will transmit about $40 \%$ in potato (HERMSEN, 1984; PELOQUin, 1982).

The objective of this study was to investigate the rate of natural numerically unreduced pollen and to elucidate the cytological mechanisms that lead to unreduced pollen formation in $P$. euphratica. Spindle orientation and other cytological characteristics were examined during the meiosis of Microspore mother cells (MMCs) in squashed anthers by indirect immunofluorescence. Based on our results on the observed spindle configurations, sporad types, and diameters of pollen grains, we propose the cytological mechanisms involved in the formation of numerically unreduced pollen and discuss the role of $2 \mathrm{n}$ pollen in triploid synthesis and polyploidy formation.

\section{Materials and Methods}

\section{Plant material}

In the spring of 2010,18 individual male trees of diploid $P$. euphratica $(2 \mathrm{n}=2 \mathrm{x}=38)$ were sampled from a natural stand grown in Ejina County, Inner Mongolia, China. 6 floral branches were collected from each sampled tree and transported to Beijing. Floral branches were detached and forced, under greenhouse conditions, at Beijing Forestry University.

\section{Pollen grain diameter analysis}

Separate pollen samples were collected from mature catkins of each clone and stored in glass bottles containing allochronic silica gel. Pollen grain were taken from the glass bottles with a dissecting needle, then mounted on a microscope slide, and stained with a $2 \%$ acetocarmine solution. The diameter of each pollen grain was assessed using an eyepiece micrometer. A total of 1150 pollen grains were measured per individual tree.

\section{Cytological analysis of male meiosis and $\chi^{2}$ test}

After the floral branches had been forced, flower buds were collected every 2-4 $\mathrm{h}$ and fixed in Carnoy's fluid (ethanol:acetic acid, 3:1) at $4^{\circ} \mathrm{C}$ until mature pollen grains appeared. For the meiosis analysis, anthers were dissected from the buds and squashed in a droplet of aceto-carmine solution (2\%) onto a microscope slide using forceps. Developing microsporocytes were characterized in detail for their spindle orientations at metaphase II and anaphase II; dyads, triads, and tetrads were determined at the sporad stage. The frequency of cells with various types of spindle arrangements at metaphase and anaphase II was determined in 558-831 MMCs. More than 500 sporads were observed to determine the proportion of tetrads, triads, and dyads at this stage.

Based on the assumption that one MMC has a certain volume and can divide into four or two parts, we can calculate a factor of appropriate 1.28 for the diameter of a diploid pollen grain compared with a haploid pollen grain (double volume of a globe), provided that the volume has not changed during the cleavage process. The largest amount of pollen grains was, in the present study, at $30 \mu \mathrm{m}$ in $P$. euphratica provided that it was the main average haploid diameter. Therefore, the observed $2 \mathrm{n}$ pollen rate was the number of large pollen grains $(\mathrm{d}>30 \mu \mathrm{m} \times 1.28=38.4 \mu \mathrm{m}) \quad / \quad 400$ observed pollen grains and the expected $2 \mathrm{n}$ pollen rate was calculated as: $(2 \times$ dyads $+1 \times$ triads $) /(2 \times$ dyads $+3 \times$ triads + $4 \times$ tetrads) under the microscope, replicated 5 times. A total of about 2000 pollen grains were used for the calculation. To assess the divergence between expected and observed $2 \mathrm{n}$ pollen frequencies, a Chi-square $\left(\chi^{2}\right)$ test was performed.

\section{Indirect immunofluorescence}

The protocol for microtubule localization during microsporogenesis was adapted from Brown and Lemmon (1992). Accordingly, the anthers were fixed for 45 min in a $4 \%$ solution of paraformaldehyde, freshly prepared in a PEM buffer (50 mM PIPES, 5 mM EGTA, 1 $\mathrm{mM} \mathrm{MgSO}_{4} ; \mathrm{pH} 6.8$ ), then permeabilized for $30 \mathrm{~min}$ in the buffer with $10 \%$ (v/v) dimethyl sulfoxide (DMSO), and finally extracted in 1\% (v/v) Triton X-100 for 15 min. After three washings in phosphate-buffered saline
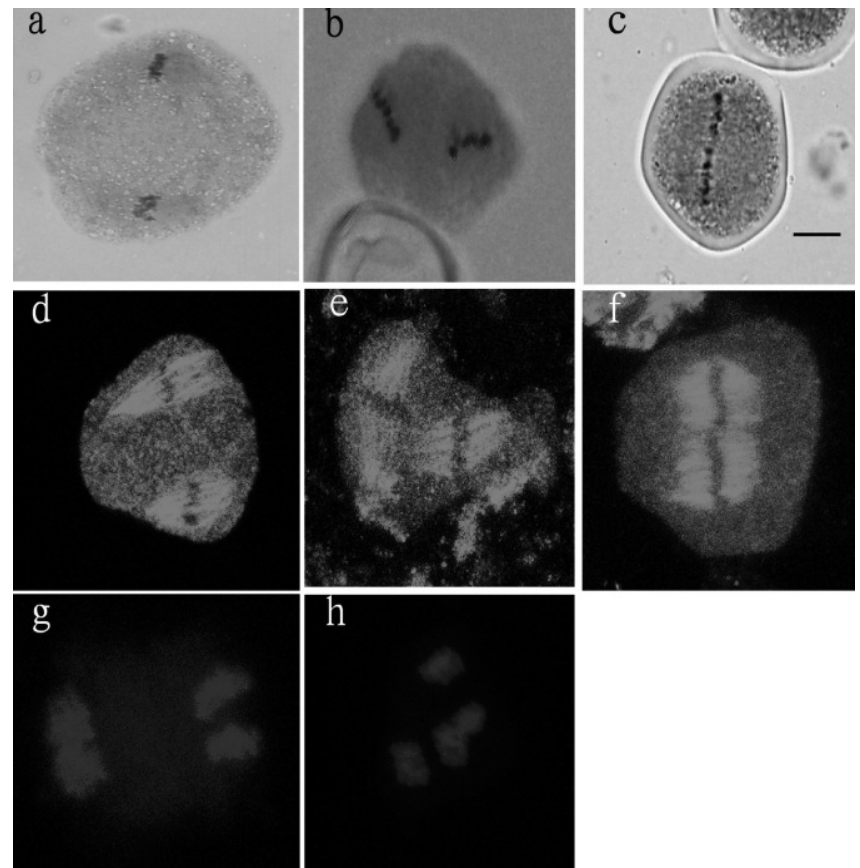

Figure 1. - Aspects of meiotic spindles at the second meiosis in microspore mother cells (MMCs) of diploid Populus euphratica. Bar: $10 \mu \mathrm{m}$. a Parallel spindle, b tripolar spindle, c fused spindle. d-f Indirect immunofluorescence study of microtubule cytoskeleton patterns at metaphase II corresponding to a-c, g-h DAPI staining of chromosome at anaphase II, which form $a-b$. 
Table 1. - Frequency of different types of spindles and sporads, expected and observed rates of $2 \mathrm{n}$ pollen grains in diploid Populus euphratica.

\begin{tabular}{|c|c|c|c|c|c|c|c|c|c|c|c|}
\hline \multirow{3}{*}{ Trees } & \multicolumn{4}{|c|}{ Different spindles at second meiotic division } & \multicolumn{4}{|c|}{ Number of different sporads } & \multirow{2}{*}{$\begin{array}{l}\text { Expected } \\
\text { rate of } 2 n\end{array}$} & \multirow{2}{*}{$\begin{array}{l}\text { Observed } \\
\text { rate of } 2 n\end{array}$} & \multirow[t]{3}{*}{$\chi^{2}$} \\
\hline & Total MMCs & Parallel & Tripolar & Fused & Total sporads & Tetrads & Triads & Dyads & & & \\
\hline & analyzed & $(\%)$ & $(\%)$ & $(\%)$ & analyzed & $(\%)$ & $(\%)$ & $(\%)$ & pollen $(\%)$ & pollen $(\%)$ & \\
\hline HY1 & 701 & 91.01 & 8.56 & 0.43 & 809 & 98.00 & 0.99 & 0.99 & 0.75 & 0.99 & \\
\hline HY2 & 558 & 85.67 & 10.75 & 3.58 & 806 & 96.15 & 0.75 & 3.10 & 1.77 & 0.97 & \\
\hline HY6 & 764 & 93.46 & 6.54 & 0 & 799 & 97.37 & 2.25 & 0.38 & 0.76 & 1.43 & \\
\hline HY7 & 723 & 94.19 & 4.15 & 1.66 & 863 & 97.33 & 1.74 & 0.93 & 0.91 & 0.49 & \\
\hline HY8 & 662 & 96.52 & 1.21 & 2.27 & 809 & 98.27 & 1.36 & 0.37 & 0.53 & 1.79 & \\
\hline HY14 & 831 & 87.01 & 12.03 & 0.96 & 781 & 90.79 & 3.58 & 5.63 & 3.85 & 4.35 & \\
\hline HY15 & 779 & 78.94 & 19.26 & 1.80 & 798 & 95.46 & 1.41 & 3.13 & 1.95 & 2.93 & \\
\hline HY16 & 741 & 93.93 & 0.67 & 5.40 & 533 & 98.31 & 1.31 & 0.38 & 0.52 & 0.97 & \\
\hline HY17 & 694 & 99.14 & 0 & 0.86 & 778 & 99.36 & 0.51 & 0.13 & 0.19 & 0 & \\
\hline HY20 & 797 & 84.31 & 10.04 & 5.65 & 812 & 93.10 & 4.56 & 2.34 & 2.36 & 3.81 & 18.71 \\
\hline HY23 & 599 & 98.00 & 0.33 & 1.67 & 813 & 96.80 & 1.23 & 1.97 & 1.31 & 4.33 & \\
\hline HY24 & 806 & 98.01 & 0.50 & 1.49 & 783 & 97.57 & 1.40 & 1.03 & 0.87 & 0.48 & \\
\hline HY25 & 773 & 96.24 & 2.85 & 0.91 & 812 & 95.07 & 3.20 & 1.73 & 1.69 & 4.37 & \\
\hline HY27 & 681 & 97.21 & 1.91 & 0.88 & 651 & 98.62 & 0.77 & 0.61 & 0.50 & 0.95 & \\
\hline HY28 & 718 & 98.88 & 0.28 & 0.84 & 787 & 98.09 & 1.14 & 0.77 & 0.67 & 0.49 & \\
\hline HY31 & 685 & 99.41 & 0.44 & 0.15 & 869 & 98.16 & 1.27 & 0.57 & 0.61 & 0 & \\
\hline HY33 & 759 & 82.87 & 3.95 & 13.18 & 796 & 94.10 & 2.63 & 3.27 & 2.35 & 2.34 & \\
\hline HY34 & 645 & 99.07 & 0.93 & 0 & 768 & 96.22 & 3.13 & 0.65 & 1.12 & 0.99 & \\
\hline Average & 717.6 & 93.19 & 4.50 & 2.32 & 781.5 & 96.60 & 1.85 & 1.55 & 1.25 & & \\
\hline
\end{tabular}

MMC, Microspore mother cell. 
(PBS; $137 \mathrm{mM} \mathrm{NaCl}, 2.7 \mathrm{mM} \mathrm{KCl}, 8 \mathrm{mM} \mathrm{Na}{ }^{2} \mathrm{HPO}_{4}$, $1.5 \mathrm{mM} \mathrm{KH} \mathrm{PO}_{4} ; \mathrm{pH} 7.4$ ), the anthers were dissected with forceps to release the meiocytes, tetrads, or young microspores. Cells were then affixed to a slide coated with $0.1 \%$ (w/v) poly-L-lysine (P1274; Sigma, St. Louis, MO) and incubated in a moist chamber with an anti-atubulin antibody (T9026; Sigma) diluted 1:200 in PBS for $1 \mathrm{~h}$ at $37^{\circ} \mathrm{C}$. Following further washing in PBS, the samples were labeled with a 1:50 (v/v) dilution of fluorescein isothiocyanate (FITC) conjugated anti-mouse immunoglobulin (Ig)G antibody (F0219; Sigma) for at least $1 \mathrm{~h}$ at $37^{\circ} \mathrm{C}$ in a moist chamber. For correlation with nuclear stages, samples were finally washed in PBS, counterstained with DAPI (40, 6-diamidino-2phenylindole) for $5 \mathrm{~min}$ and mounted in a Prolong Gold antifade reagent (P36934; Invitrogen, Carlsbad, CA). The preparations were observed using a fluorescence microscope (model BX51; Olympus, Japan) and photographed with a CCD camera (model DP70; Olympus).

\section{Results}

\section{Cytological characteristics of male meiosis}

The fist meiotic division of all 18 individual trees was found to progress normally, but a number of cytological events occurred during the second meiotic division that resulted in $2 \mathrm{n}$ pollen formation. At metaphase and anaphase II, the orientations of the spindles of homologous chromosomes were parallel, tripolar, and fused, as observed in pollen specimens that had been stained in the aceto-carmine solution (Fig. 1a-c). There was a predominance of parallel spindles in all 18 individual trees of $P$. euphratica. Tripolar and fused spindles were observed frequently in trees HY14 and HY20, respectively, whereas the tree HY33 was the highest total producer of these two abnormal spindles (see Table 1). The angle of the meiotic spindles at anaphase II was also arranged from parallel to tripolar, which was illustrated by the position of the four-sister chromosome
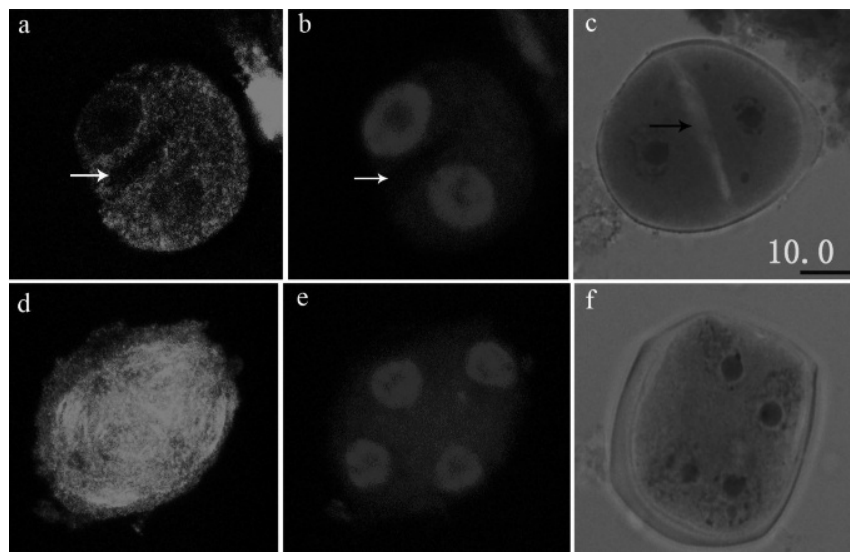

Figure 2. - Microtubules arrays, chromatin location, and cytokinesis at meiosis of MMCs in diploid P. euphratica. a, d Microtubules, b, e DAPI staining of chromosome, $\mathbf{c}, \mathbf{f}$ Microtubuless, acetocarmine staining of chromosome. Bar: $10 \mu \mathrm{m}$. a-c The beginning of the furrow (arrow), showing the occurrence of simultaneous cytokinesis. d-f Cytokinesis after meiosis, with radial microtubule systems (RMSs) surrounding each nucleus.
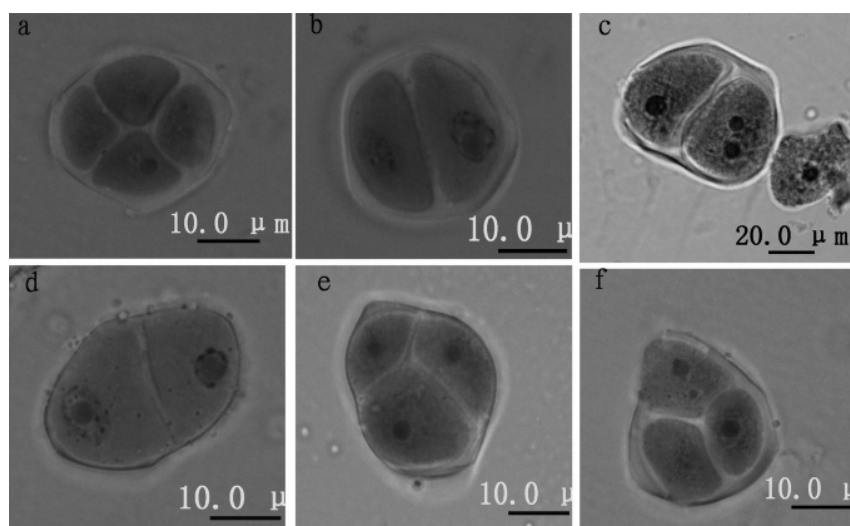

Figure 3. - Different types of sporads at tetrad stage of diploid $P$. euphratica. a A tetragonal tetrad with four reduced microspores. b A dyad with two nucleoli of nearly the same size being included in each of the two restitution nuclei. c A dyad with two restitution nuclei, but one of them containing two nucleoli. d A dyad with one larger microspore and one smaller microspore, each of the two dyad microspores containing one restitution nucleus. e A triad with the large microspore containing one restitution nucleus and two smaller microspores each containing one nucleus. f A triad with one restitution nucleus containing two nucleoli.

complement (Fig. 1g, h). Indirect immunofluorescence microscopy of microtubule cytoskeleton patterns during meiosis revealed that the poles of the tripolar and fused spindles in some MMCs were joined at one or two axes of the cell (Fig. 1e,f).

\section{Cytokinesis and abnormal sporads formation}

Prematural cytokinesis after the first meiotic division was observed (Fig. $2 a-c$ ). The formation of cleavage furrows pinpointed the beginning of the simultaneous cytokinesis, and this cleavage formation progressed from the center to the periphery of the dividing cells (Fig. 2c).

Phragmoplasts were not found at telophase II, but radial microtubule systems (RMSs), which expanded from the sister nuclei and non-sister nuclei, were observed (Fig. 2d-f) in MMCs with parallel spindles. Generally, meiosis and cytokinesis resulted in the production of tetragonal tetrads with four reduced microspore (Fig. 3a), but different types of dyads $(F i g .3 b-d)$ and triads (Fig. 3e, f) with one or two unreduced microspores were found at the tetrad stage. These unreduced microspores had a restitution nucleus with one (Fig. $3 b, d, e)$ or two nucleoli (Fig. $3 c, f)$. The triads presented asymmetric cytokinesis (Fig. 3e,f), but an unbalanced divisiong of cytoplasm also occurred in the dyads. The frequency of triads, dyads, and tetrads was calculated for 18 clones of $P$. euphratica with staminate buds at the end of telophase II (Table 1). Of the more than 530 spores counted in randomly selected anthers from each sampled tree, the frequency of triads, dyads, and tetrads ranged from $0.51 \%$ to $4.56 \%, 0.37 \%$ to $5.63 \%$, and $90.79 \%$ to $99.36 \%$, respectively. The correlation analysis showed a significant association $(r=0.68$, $P<0.05$ ) only between the tripolar spindles and dyads (data not show), but the association of parallel and fused spindles between dyads and triads were not significant. 


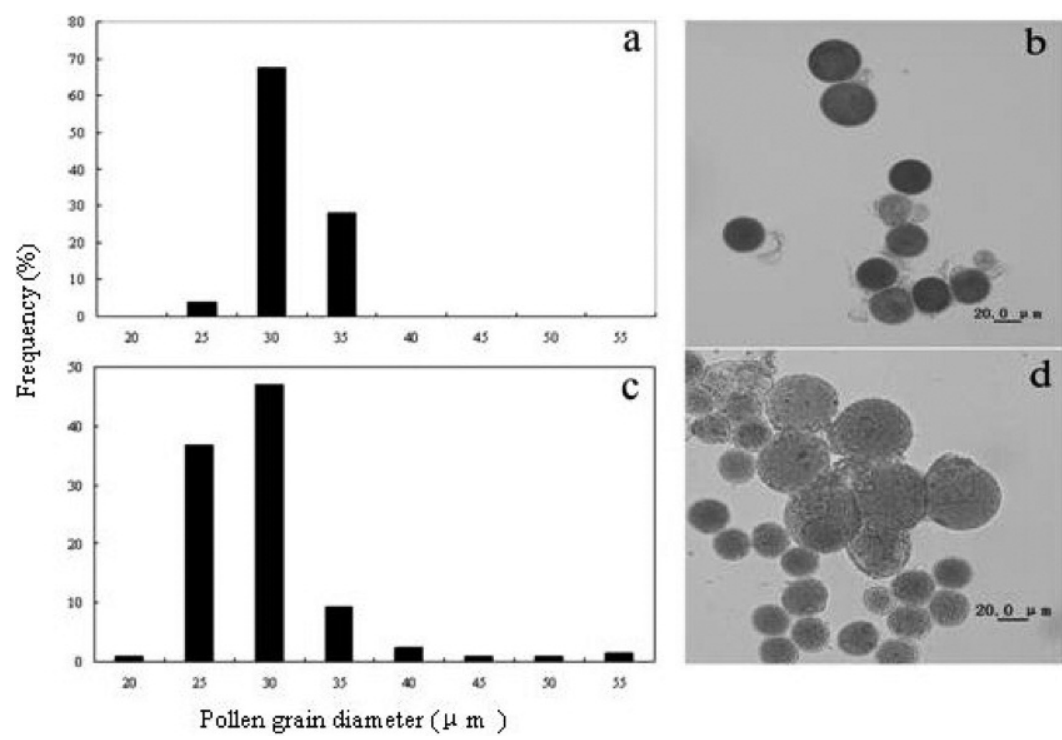

Figure 4. - Frequency distribution of pollen diameter in tree HY17 (a, b) and tree HY25 (c, d) of diploid P. euphratica. Bar. $20 \mu \mathrm{m}$.

\section{Rates and its cytological determination of $2 n$ pollen formation}

The rates of $2 \mathrm{n}$ pollen of 18 sampled trees in diploid P. euphratica were presented in Table 1. Among the 18 genotypes, 16 genotypes produced $2 \mathrm{n}$ pollen and two genotypes (trees HY17 and HY31) produced only normal $\mathrm{n}$ pollen. In all $2 \mathrm{n}$ pollen producers, the rates of $2 \mathrm{n}$ pollen varied from 0 to $4.37 \%$, and the rates of $2 \mathrm{n}$ pollen of trees HY25, HY14, HY23 and HY20 are higher than that of other producers.

Normal microsporogenesis in P. euphratica is simultaneous cytokinesis, and formed a tetrad in a tetrahedral arrangement (Fig. 3a). However, parallel spindles, tripolar and fused spindles (Fig. 1a-c), and premature cytokinesis II (Fig. 2a-c) were observed during the meiotic division. Tripolar and fused spindles were the variations of parallel spindles and could result in triads and dyads, respectively. Premature cytokinesis II could result in dyads.

The abnormal rates of meiosis in $P$. euphratica were examined among more than 500 meiotic cells. The expected $2 \mathrm{n}$ pollen rates were accordant with the observed $2 \mathrm{n}$ pollen rates by $\chi^{2}$ test (Table 1 ).

\section{Pollen grain size distribution}

The fertile pollen grains were stained deeply by the $2 \%$ aceto-carmine solution, while the aborted ones could either not be stained or could only be stained lightly. Therefore, it was very easy to measure the pollen grains under a light microscope. The size of the pollen grains ranged from $18.4 \mu \mathrm{m}$ to $53.5 \mu \mathrm{m}$ among the 18 individual trees of diploid $P$. euphratica. However, the pollen grain-size distribution of the 18 individual trees was different. The diameters of the pollen grains of tree HY17 from $22.6 \mu \mathrm{m}$ to $34.8 \mu \mathrm{m}$ (Fig. $4 a, b$ ), but the largest range was found for tree HY25, from $19.6 \mu \mathrm{m}$ to $53.5 \mu \mathrm{m}$ (Fig. 4c, d).

\section{Discussion \\ Pollen grain size and ploidy level}

In many plants, a positive correlation between pollen size and their ploidy level had often been seen (JACOB and Pierret, 2000). Because the diameter of pollen grains may become larger due to the doubling of chromosome numbers, pollen size has been widely used as an indicator of $2 \mathrm{n}$ male gametes (VEILLEUX et al., 1982). ORJEDA et al. (1990) found that the $2 \mathrm{n}$ pollen of the sweet potato (Ipomoea trifida) was about $30 \%$ larger than normal pollen.

SEITZ (1954) observed the presence of giant pollen grains in the anomalous androgynous flowers of the grey poplar ( $P$. canescens) and, subsequently the formation of triploids after self-pollination. The pollen grain of the triploid aspen ( $P$. tremuloides) was found to be between 37 and $44 \mu \mathrm{m}$ in diameter, and tetraploid hybrids were obtained by crossing pollen of the triploid tree with a diploid female (Winton and Einspahr, 1970). The induced unreduced pollen of $P$. tomentosa was distinctly larger than normal pollen, which can be beyond $37 \mu \mathrm{m}$ in diameter (ZHU et al., 1998). Based on these studies, the diameter of unreduced pollen grains in the poplar genus is at least $37 \mu \mathrm{m}$ in diameter. In the present study, pollen grains with diameters $>38.4 \mu \mathrm{m}$ were frequently observed at different frequencies among all 18 trees (see Table 1), and giant pollen grains were also observed in some trees. These giant pollen grains are considered to be either $3 \mathrm{n}$ or $4 \mathrm{n}$ pollen grains (TAVOLETTI et al., 2000; CAMADRO et al., 2008), which would indicate that over-diploid pollen grains do occur in these trees of P. euphratcia.

\section{Cytological mechanism of $2 n$ pollen formation}

Abnormal meiotic division led to $2 \mathrm{n}$ pollen formation. These abnormal meiotic phenomena were mainly owing to the following: (1) Synaptic mutant: in potato, a synap- 
tic mutant resulted in the homologous chromosomes failing to pair and cross-over rate reduction (DoucHES, 1988). This mechanism can form FDR 2 n gametes finally. (2) Abnormal spindles orientation: Parallel, fused, and tripolar spindles are the three main types of spindle configurations that have been showed in plants that produce 2n pollen (MoK and Peloquin, 1975; VeIlleuX, 1985; BRETAGNOLle and ThOMPSON, 1995; RAMSEY and Schemske, 1998; ZHANG et al., 2009; ZHANG and KANG, 2010; Silva et al., 2011). The parallel spindles resulted in two FDR $2 \mathrm{n}$ pollens. The fused spindles led a mother cell to form a dyad and then two FDR $2 \mathrm{n}$ pollens were produced, and the tripolar spindles formed one FDR 2n pollen and two $1 \mathrm{n}$ pollen. (3) Nuclear fusion: at anaphase II and telophase II, two adjacent daughter nuclei fused together to form two FDR 2n pollen (CHEN, 1997). (4) Premature cytokinesis: cytoplasm divided early after meiosis I; however, sister chromatids divided normally. The result of this mechanism is the formation of two SDR 2n pollens (MoK and PELOQUin, 1975). Many plants have more than one of the above mechanisms to form $2 \mathrm{n}$ pollen. Potato has parallel spindle, fused spindle, premature cytokinesis- 1 and premature cytokinesis2 (OliveIRA, 1995). Persimmon (Diospyros kaki L.) has parallel spindle, fused spindle and tripolar spindle (TANG, 2002). The parallel spindle, fused spindle, tripolar spindle and premature cytokinesis- 2 were observed in $P$. tomentosa (ZHANG and KANG, 2010), $P$. $\times$ euramericana and $P . \times$ popularis (ZHANG et al., 2009).

In this study, we observed four types of abnormalities: parallel spindle, fused spindle, tripolar spindle and premature cytokinesis in $P$. euphratica. These abnormalities can be classified as having abnormal spindle fiber orientation during meiosis II and early division of cytoplasm after meiosis I. The statistical analysis of abnormal spindles and sporads indicated that the frequent parallel spindles may lead to triads and dyads formation at the tetrad stage. This could be owing to the gathering of one or two non-sister sets of chromosomes at telophase II, which results in two nuclear cytoplasmic domains in late-telophase cells and the division of MMCs into either triads or dyads. The dyad developed into two $2 \mathrm{n}$ pollens and the triad grew into one $2 \mathrm{n}$ pollen and two $1 \mathrm{n}$ pollens. The $2 n$ pollen formed in these ways was genetically equivalent to FDR. Premature cytokinesis II could result in dyads. The $2 \mathrm{n}$ pollen grains formed via this abnormal approach were genetically equivalent to SDR.

Due to simultaneous cytokinesis, the RMSs emanating from daughter nuclei define the spore domains and determine the placement of intersporal walls (BROWN and LEMMON, 1992). The establishment of spore domains via RMSs can account for both the regular and irregular patterns of tetrad formation (BROWN and LEMMON, 1991). In $P$. euphratica, the position of the sister nuclei confined the RMSs as well as the plane of division.

\section{Implications for polyploidy breeding}

As a genetic consequence, two types of $2 n$ gametes can be obtained, corresponding to FDR and SDR. Due to the equational division of sister chromatids, FDR gametes contain an equal number of parental chromosomes. On the other hand, in the case of SDR, sister chromosomes move to the same daughter cell. Therefore, FDR gametes contain maximum heterozygosity and SDR gametes display maximum homozygosity in the absence of crossing-over (HeRmsen, 1984; Veilleux, 1985; PeloQUIN et al., 1999). Consequently, FDR gametes are particularly valuable in plant sexual polyploidization breeding programs (BRETAGNolle and Thompson, 1995). The unreduced pollen resulting from abnormal spindles is genetically equivalent to the FDR mechanism, while that from premature cytokinesis is genetically equivalent to SDR (RAMANNA, 1979). In the present study, the unreduced pollen of $P$. euphratica caused by abnormal spindles is genetically equivalent to the FDR mechanism, while that from premature cytokinesis is genetically equivalent to SDR. Because our cytological analysis was performed only to determine the expected heterozygosity of unreduced gametes, further investigations are required to reveal the actual level of heterozygosity of the unreduced gametes and their contribution to the progeny.

Natural triploids often occur in the genus Populus, and have been widely used in forestation and forestry production (ZHANG et al., 2004). These natural triploid hybrids are presumably due to the production of numerically unreduced pollen (ZHU et al., 1998; ZHANG et al., 2004). Studies on the genetic control of unreduced pollen and the techniques for discovering producers of a higher frequency of unreduced pollen will provide a platform for the genetic improvement and triploid breeding of P. euphratica.

\section{Acknowledgements}

We thank Ejina bureau of foresty in Inner Mongolia for providing the plant materials. The research was supported by the Fundamental Research Funds for the Central Universities (NO.YX2013-07) and the National Natural Science Foundation of China (No. 31370659).

\section{References}

Akutsu, M., S. Kitamura, R. Toda, I. Miyajima and K. OKAZAKI (2007): Production of $2 \mathrm{n}$ pollen of Asiatic hybrid lilies by nitrous oxide treatment. Euphytica 155: 143-152.

Bradshaw, H. and R. Stettler (1993): Molecular genetics of growth and development in Populus. I. Triploidy in hybrid poplars. Theor Appl Genet 86: 301-307.

Bretagnolle, F. and J. Thompson (1995): Gametes with the somatic chromosome number: mechanisms of their formation and role in the evolution of autopolyploid plants. New Phytol 129: 1-22.

Brown, R. and B. LEMmon (1991): Pollen development in orchids 2. The cytokinetic apparatus in simultaneous cytokinesis. Protoplasma 163: 9-18.

Brown, R. and B. LEMmon (1992): Control of division plane in normal and griseofulvin-treated microsporocytes of Magnolia. J Cell Sci 103: 1031-1038.

Camadro, E., S. Saffarano, J. Espinillo, M. Castro and P. Simon (2008): Cytological mechanisms of $2 \mathrm{n}$ pollen formation in the wild potato Solanum okadae and pollen-pistil relations with the cultivated potato, Solanum tuberosum. Genet Resour Crop Evol 55: 471-477. 
CARPuto, D. and A. BARONE (2005): Ploidy level manipulations in potato through sexual hybridisation. Ann Appl Biol 146: 71-79.

Chen, C., D. Sleper, S. Chao, G. Johal, C. West and P. MADISON (1997): RFLP detection of 2n pollen formation by first and second division restitution in perennial ryegrass. Crop Sci. 37: 76-80.

Conicella, C., A. Capo, M. Cammareri, A. Errico, N. ShamINA and L. MONTI (2003): Elucidation of meiotic nuclear restitution mechanisms in potato through analysis of microtubular cytoskeleton. Euphytica 133: 107-115.

d'Erfurth, I., S. Jolivet, N. Froger, O. CAtrice, M. NovatchKova and M. SIMON (2008): Mutations in AtPS1 (Arabidopsis thaliana Parallel Spindle 1) lead to the production of diploid pollen grains. PLoS Genet 4(11): 1000274.

Den NiJs, T. and S. Peloquin (1977): 2n gametes in potato species and their function in sexual polyploidization. Euphytica 26: 585-600.

Douches, D. and C. QuiRos (1988): Genetic recombination in a diploid synaptic mutant and a solanum tuberosum $\times$ S. chacoense diploid hybrid. Heredity, 83: 346-349.

Genualdo, G., A. Errico, A. Tiezzi and C. Conicella (1998): a-Tubulin and F-action distribution during microsporogenesis in a $2 \mathrm{n}$ pollen producer of Solanum. Genome 41: 636-641.

HARLAN, J. and J. De Wet (1975): On O*. Winge and a prayer: the origins of polyploidy. Bot Rev 41: 361-390.

HERMSEN, J. (1984): Mechanisms and genetic implications of 2n-gametes formation. Iowa State J. Res. 58: 421-434.

JacoB, Y. and V. Pierret (2000): Pollen size and ploidy level in the genus Rosa. Acta Hortic 508: 289-292.

JAUHAR, P. (2003): Formation of $2 n$ gametes in durum wheat haploids: sexual polyploidization. Euphytica 133 81-94.

Lim, K., M. Ramanna, J. DE Jong, E. Jacobsen and J. van TUYL (2001): Indeterminate meiotic restitution (IMR): a novel type of meiotic nuclear restitution mechanism detected in interspecific lily hybrids by GISH. Theor Appl Genet 103: 219-230.

Mendiburu, A. O., S. J. Peloquin and D. W. S. MoK (1974) Potato breeding with haploids and $2 \mathrm{n}$ gametes. In: KASHA, K. (ed) Haploids in higher plants. University of Guelph, Guelph.

MoK, D. and S. Peloquin (1975): Three mechanisms of $2 \mathrm{n}$ pollen formation in diploid potatoes. Can J Genet Cytol 17: 217-225.

Muntzing, A. (1936): The chromosomes of a giant Populus tremula. Hereditas 21: 383-393.

OLIVEIRA, M. (1995): Mechanism of $2 \mathrm{n}$ pollen formation in diploid Solanum tuberosum $\times S$. chacoense Bitt hybrid clones. Brazilian J. Genet. 18: 445-450.

OrJedA, G., R. FReYRe and M. IwaNAGa (1990): Production of $2 \mathrm{n}$ pollen in diploid Ipomoea trifida, a putative wild ancestor of sweet potato. J Hered 81: 462-467.

PELOQUIN, S. (1982): Meiotic mutants in potato breeding. Stadler Genet. Symp. 14: 99-109.

Peloquin, S., L. Bioteux and D. Carputo (1999): Meiotic mutants in potato: Valuable variants. Genetics, 153: 1493-1499.
RAMANNA, M. (1979): A re-examination of the mechanisms of $2 \mathrm{n}$ gamete formation in potato and its implications for breeding. Euphytica 28: 537-561.

RAMSEY, J. (2007): Unreduced gametes and neopolyploids in natural populations of Achillea borealis (Asteraceae). Heredity 98: 143-150.

RAMseY, J. and D. Schemske (1998): Pathway, mechanisms, and rates on polyploid formation in flowering plant. Annu Rev Ecol Syst 29: 467-501.

RIEseberg, L. H. and J. H. Willis (2007) Plant speciation. Science 317: 910-914. doi:10.1126/science.1137729

SEITZ, F. (1954): The occurrence of triploids after self-pollination of anomalous androgynous flowers of a grey poplar. Z Forstgenet 3(1): 1-6.

Silva, N., A. Mendes-Bonato, J. Sales and M. Pagliarini (2011): Meiotic behavior and pollen viability in Moringa oleifera (Moringaceae) cultivated in southern Brazil. Genet. Mol. Res. 10(3): 1728-1732.

Sugiura, A., T. Ohkuma, Y. Choi, R. TAo and M. TAmura (2000): Production of nonaploid $(2 \mathrm{n}=9 \mathrm{x})$ Japanese persimmons (Diospyros khaki) by pollination with unreduced $(2 \mathrm{n}=6 \mathrm{x})$ pollen and embryo rescue culture. J Am Soc Hortic Sci 125(5): 609-614.

TANG, X. and Z. LUO (2002): Cytology of $2 \mathrm{n}$ pollen formation in nonastringent persimmon. Sci. Agric. Sin. 35 : 585-588.

Tavoletti, S., P. Pesaresi, G. Barcaccia, E. Albertini and F. VERONESI (2000): Mapping the jp (jumbo pollen) gene and QTLs involved in multinucleate microspore formation in diploid alfalfa. Theor Appl Genet 101: 372-378.

VAN TUYL, J. and K. LIM (2003): Interspecific hybridization and polyploidisation as tools in ornamental plant breeding. Acta Hortic 612: 13-22.

Veilleux, R. (1985): Diploid and polyploid gametes in crop plants: Mechanisms of formation and utilization in plant breeding. Plant Breed Rev 3: 253-288.

Veilleux, R., N. McHale and F. Lauer (1982): 2n gametes in diploid Solanum: frequency and type of spindle abnormalities. Can J Genet Cytol 24: 301-314.

WinTon, L. and D. EinspaHR (1970): Tetraploid aspen production using unreduced triploid pollen. For Sci 16: 180-182.

ZHANG, J., Z. WeI, D. LI and B. LI (2009): Using SSR markers to study the mechanism of $2 \mathrm{n}$ pollen formation in Populus $\times$ euramericana (Dode) Guinier and $P . \times$ popularis. Ann. For. Sci. 66(506): 1-10.

Zhang, S., L. Qi, C. Chen, X. Li, W. Song and R. Chen (2004): A report of triploid Populus of the section Aigeiros. Silvae Genet 53: 69-75.

ZHANG, Z. and X. KANG (2010): Cytological characteristics of numerically unreduced pollen production in Populus tomentosa Carr. Euphytica 173: 151-159.

Zhang, Z., X. Kang, P. Zhang, Y. Li and J. WANG (2007): Incidence and molecular markers of $2 \mathrm{n}$ pollen in $\mathrm{Popu}$ lus tomentosa Carr. Euphytica 154: 145-152.

ZHU, Z. (2006): Genetic improvement of Populus tomentosa. Chinese Forestry Press, Beijing. pp 155-220.

ZHU, Z., X. KANG and Z. ZHANG (1998): Studies on selection of natural triploid of Populus tomentosa. Sci Silvae Sin 34: 22-31. 